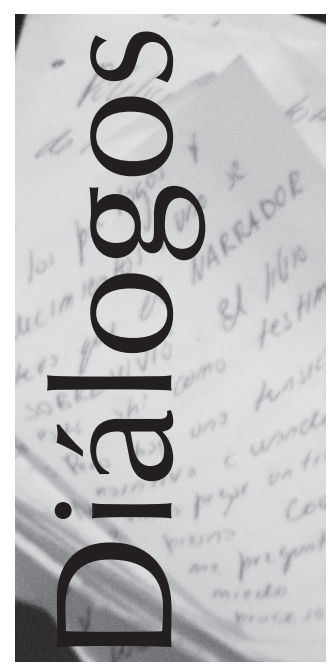

\title{
La vulnerabilidad estructural y las nuevas perspectivas en medicina social sobre la salud de los migrantes: entrevista a James Quesada y Seth M. Holmes
}

\author{
Structural vulnerability and new perspectives in social \\ medicine on the health of immigrants: Interview with \\ James Quesada and Seth M. Holmes
}

Carlos Piñones Rivera', James Quesada², Seth M. Holmes ${ }^{3}$

'Psicólogo, Doctor en Antropología Médica. Investigador del Instituto de Estudios Internacionales, Universidad Arturo Prat, Iquique, Chile. $\square$ (iD)

${ }^{2}$ Doctor en Antropología Médica. Profesor, César Chavez Institute, Department of Anthropology, San Francisco State University, San Francisco, California, EEUU. $\triangle$ (iD)

'Médico, Doctor en Antropología Médica. Profesor Asociado UC Berkeley's Division of Society and Environment, UCSF-Berkeley's Joint Program in Medical Anthropology, University of California, California EEUU. $\square$ (iD)
RESUMEN Desde hace una década, varios autores anglófonos, centrados principalmente en el análisis e intervención de los procesos de determinación social de la salud de los migrantes, forjaron el concepto de vulnerabilidad estructural, como una forma de combatir el individualismo, el biologismo, la invisibilización de los procesos de determinación estructural y la culpabilización de las víctimas. Siendo parte de las contribuciones históricas de la medicina social, los actuales desarrollos de la aproximación sobre la vulnerabilidad estructural han quedado desconectadas de las discusiones del movimiento de la salud colectiva y la medicina social latinoamericana en general, entre otras razones, por las barreras lingüísticas asociadas a la escasez de sus publicaciones en español. La presente entrevista, realizada a dos de sus principales representantes, indaga los orígenes históricos de dicha aproximación y busca explorar las contribuciones específicas que hoy está realizando, como una forma de acercarlas a los lectores de habla hispana, favoreciendo el diálogo con las propuestas de medicina social latinoamericanas.

PALABRAS CLAVES Vulnerabilidad en Salud; Medicina Social; Inmigración; Antropología Médica; Determinantes Sociales de la Salud.

\begin{abstract}
A decade ago, a number of English-speaking authors focused mainly on the analysis and intervention of processes of social determination of health of migrants developed the concept of structural vulnerability as a way to combat individualism, biologism, the invisibilization of processes of structural determination and the blaming of victims. As part of the historical contributions of social medicine, the current developments of the structural vulnerability approach have been disconnected from the discussions of the collective health movement and Latin American social medicine in general, among other reasons due to linguistic barriers associated with the scarcity of publications in Spanish. The present interview, conducted with two of the primary representatives of the structural vulnerability approach, investigates its historical origins and seeks to explore the specific contributions that are being made today, as a way to bring them closer to Spanish-speaking readers and so enable dialogue with the proposals of Latin American social medicine.
\end{abstract}

KEY WORDS Vulnerable Populations; Social Medicine; Human Migration; Medical Anthropology; Social Determinants of Health. 


\section{PRESENTACIÓN}

La vulnerabilidad estructural ha sido definida como una posicionalidad que impone sufrimiento físico-emocional a colectivos e individuos específicos de manera estructurada, producto de la explotación económica basada en la clase y la discriminación cultural, género/sexual y racial, así como en procesos complementarios de formación de subjetividad depreciada ${ }^{(1)}$. Desarrollado por James Quesada y otros autores contemporáneos anglófonos, el concepto ha tenido un impulso sin par en manos de algunos investigadores en California, asociados al Berkeley Center for Social Medicine (James Quesada y Seth Holmes), a la University of California Los Ángeles (Phillipe Bourgois), a la University of California San Francisco (Kelly Knight), a la San Francisco State University (Félix Kury) y al Structural Competency Working Group (Joshua Neff, Shirley Strong) -por nombrar algunos de los más representativos- y se está expandiendo vigorosamente al resto del mundo, Ilegando a constituir una perspectiva en el abordaje de la salud de los migrantes y de los "condenados de la tierra" en general, que pone un decidido énfasis en la importancia de los procesos de determinación económico-políticos y extrae las consecuencias prácticas del reconocimiento de dichos procesos, en diálogo con las teorizaciones previas o paralelas de la medicina social en Europa, América Latina y el mundo.

Los desarrollos sobre la vulnerabilidad estructural surgen en el trasfondo de una decidida crítica a las aproximaciones socioculturalistas $^{(1,2)}$ y tienen como antesala los trabajos de la antropología médica crítica estadounidense (Baer, Singer, etc.), los de la antropología médica crítica interpretativista (Scheper-Hughes, Bourgois, etc.), aquellos sobre la violencia estructural (Galtung, Farmer, etc.) y los del sufrimiento social (Kleinman, Das, etc.), entre otros. Comparten el diagnóstico de que en los casos de los problemas de salud de los migrantes (pero no solo de ellos), siempre se trata de "más que cultura", según sintetizó Villa-Torres et al.(2).
También dialogan con las propuestas de la teoría crítica de la raza ${ }^{(3,4,5,6)}$ y las contribuciones feministas desde el concepto de interseccionalidad $^{(7,8)}$. Desde todos estos frentes, buscan abordar de manera renovada la determinación de clase, género y étnico-racial de la salud, poniendo énfasis en el carácter estructural de estas distintas "opresiones conjugadas $^{\prime \prime(9)}$.

Estos trabajos están movidos, en buena medida, por la sentida necesidad de desarroIlar una aproximación, un lenguaje y unos métodos, que permitan establecer un diálogo fructífero con los profesionales de la salud y sean relevantes para la atención clínica. Como especificaron Quesada et al. ${ }^{(1)}$ :
...nuestro requisito aquí es que la vul- nerabilidad debe abordarse no solo en el ámbito político, sino también en el encuentro clínico [...] necesitamos responder al desafío de la antropolo- gía médica crítica para ir más allá de la academia y proponer intervenciones prácticas que tengan consecuencias inmediatas. (traducción propia).

Esto, que podríamos Ilamar una relación pragmático-crítica con la clínica (y no solo con la prevención, la promoción, los aspectos administrativos-burocráticos o la abogacía), constituye, a nuestro parecer, uno de sus impulsos distintivos, dando origen a una propuesta relativamente sistemática, que empuja hacia el desarrollo de competencias estructurales (en vez o a la par de las "culturales") en el personal de salud ${ }^{(3,10,11,12)}$.

La presente entrevista, realizada a James Quesada y Seth M. Holmes por Carlos Piñones Rivera el 8 de octubre del 2018, en su calidad de visiting scholar en el Institute for the Study of Societal Issues del Berkeley Center for Social Medicine, en el marco del Fondecyt Postdoctorado, proyecto $N^{\circ} 3180173$, indaga los orígenes históricos de dicha aproximación y busca explorar las contribuciones específicas, como una forma de acercarlas a la audiencia de habla hispana, y favorecer así el diálogo con las propuestas de medicina social latinoamericanas. 


\section{DIÁLOGO}

Carlos Piñones Rivera: La primera pregunta es para ti, Jim. Tu trabajo está íntimamente vinculado con una reflexión profunda sobre la violencia estructural ¿Puedes decirnos cómo llegaste al concepto de vulnerabilidad estructural?

James Quesada: Bueno, la primera vez que escuché "vulnerabilidad estructural" fue de un antropólogo mexicano, Daniel Hernández Rosete ${ }^{(13)}$... A principios de la década del 2000, estábamos trabajando en un estudio binacional de trabajadores migrantes. Recuerdo que le pregunté sobre el término y aún estaba en formación, aún era embrionario, por la forma en que hablaba al respecto. Entonces, la forma en que yo lo entendí, fue realmente tomando en cuenta todos los factores sociales y las fuerzas estructurales que afectan, que dan forma e influyen en la vida de las personas, a menudo en su detrimento.

Tiene algo que ver pero, a la vez, es algo distinto a la competencia cultural, si la pensamos como aquello de la cultura que se debe tener en cuenta para comprender el bienestar o la mala salud de una persona. Las fuerzas estructurales parecen ser aún más importantes de considerar y particularmente con la población con la que estábamos trabajando, trabajadores latinos indocumentados que no tienen un ingreso estable, la residencia donde viven es problemática, trabajan como jornaleros [day laborers], que no es en ningún sentido un trabajo estable. Están mirando sobre sus hombros porque "la migra" [Servicio de Inmigración y Aduanas de Estados Unidos] puede capturarlos, y queríamos tener en cuenta todos estos problemas con los que se enfrentan a diario. Entonces, al principio todo esto surgió al tratar con trabajadores agrícolas migrantes, pero cuanto más lo pensamos es aplicable a todo tipo de personas en toda la sociedad y en muchas poblaciones diferentes y no solo en una de las poblaciones que creo que es la más vulnerable hoy en día en este país, que es la población latina indocumentada.
Carlos: He leído en tu presentación, en la página web de la universidad, que te alineas con una tradición crítica de antropología médica. ¿Qué había de insatisfactorio en la producción de la antropología médica crítica que te llevó a desarrollar tu propio enfoque Ilamado "vulnerabilidad estructural"?

James: Creo que la antropología médica crítica trata de considerar seriamente toda la historia de la teoría crítica para examinar el mundo que nos rodea. Significa tomar en serio la economía-política, tomar en serio la historia, tratar realmente de comprender el fundamento social y la distribución social de la mala salud y del bienestar. La biomedicina y los enfoques biomédicos, si bien son absolutamente importantes, y deben tenerse en cuenta, no pueden verse o aislarse de estos otros factores. Entonces, el enfoque de la antropología médica crítica consiste en tratar de tomar en cuenta, de manera más sistemática, todas estas otras fuerzas, los factores que dan forma a la vida de las personas y a las consecuencias y resultados, sean estos positivos o negativos.

Seth Holmes: Dime si estoy entendiendo bien Jim... parece que, en parte, lo que estás diciendo es que la "vulnerabilidad estructural" es un concepto que encaja bien en la historia de la antropología médica crítica.

James: Sí, eso.

Seth: Desde mi perspectiva, como antropólogo médico y como médico, una de las cosas que tú, Daniel Hernández Rosete, Philippe Bourgois y Laurie Hart hicieron, fue abordar la forma en que la vulnerabilidad (tanto dentro de la sociedad en general, como especialmente dentro de las profesiones de la salud, la salud pública y la biomedicina) es entendida como un resultado de las características demográficas, de las características individuales o de las elecciones individuales, los comportamientos, las creencias sobre la salud... y lo que ustedes hicieron fue hacer comprensible una idea exactamente opuesta: que esa vulnerabilidad es, en gran parte y de 
forma importante, producida por estructuras de dominación, estructuras de extracción, estructuras de explotación, quién posee el capital, y cómo eso es racializado, generizado [gendered] y relacionado con el estatus ciudadano, en múltiples maneras. Y parte de lo que valoro de la vulnerabilidad estructural es que ha sido útil no solo para los cientistas sociales de la salud, sino que mucha gente ha comenzado a usar ese marco, siendo relativamente comprensible para los profesionales de la salud, de una manera que realmente contradice la suposición de que la vulnerabilidad es una cosa individual.

James: Sí, una de las cosas que creo que está infrateorizada y que realmente no se piensa en serio es que cuando consideramos la salud, tendemos a biologizarla o psicologizarla y todo el campo de lo social es algo asumido, o secundario, o que ni siquiera se tiene en cuenta. Y creo que la dimensión social, la producción y distribución social de la mala salud debe considerarse por derecho propio. Hablamos de un modelo biopsicosocial, pero lo social generalmente se deja de lado, o es secundarizado o minimizado en términos de su importancia. No estoy tratando de decir que lo social predomina, lo que sugiero es que debe tomarse muy en serio, ese es el primer punto.

El segundo punto es alejarse de la estigmatización y culpabilización de la víctima, culpar a la gente por sus propios comportamientos. Y ahí entra la psicología, donde a menudo el tipo de intervenciones que se producen en entornos de salud se orienta a que los pacientes y los clientes tienen que recalibrar y cambiar sus comportamientos para maximizar la salud, pero sin tener en cuenta todas las condiciones y circunstancias sociales que podrían impedir que las personas sean más responsables.

Sin embargo, lo que creo que es importante acerca de la vulnerabilidad estructural es que realmente debe contribuir a reformular la forma en que ofrecemos atención médica. Es bueno que hagamos diagnósticos, que entendamos los dilemas y las deficiencias que tiene la gente, por qué no pueden obtener el medicamento que necesitan, por qué no tienen una dieta más nutritiva, etcétera, etcétera. Podemos averiguar, diagnósticamente, cuáles son sus necesidades y condiciones sociales, pero ¿qué se supone que deben hacer los médicos y los profesionales de la salud con esa información? Y ese es el desafío... porque creo que lo que la vulnerabilidad estructural provoca es hacernos repensar qué es realmente la atención médica y qué son las intervenciones médicas. Entonces, de nuevo, para volver a la antropología médica crítica, cuando pienso en la salud, no puedo evitar pensar en cómo incluso los grandes médicos que se preocupan mucho por sus pacientes, muchas veces se encuentran en instituciones y tienen que adherirse a prácticas institucionales que limitan la eficacia de lo que pueden hacer. La vulnerabilidad estructural no es solo para usos de diagnóstico, sino también para desafiar a la biomedicina a pensar realmente como institucionaliza las prácticas.

Seth: En relación con lo que mencionaste de la vulnerabilidad estructural y la atención médica, nos recuerda que hay tanta evidencia de la determinación social de la salud, de la producción estructural de vulnerabilidad, que la respuesta apropiada -o la respuesta más apropiada-, no es solo intervenir en el individuo, ya sea a través de prescripciones clínicas o a través de la salud pública, programas de educación para la salud, sino que de manera importante, quizás incluso más importante, es intervenir, en primer lugar, en las estructuras sociopolíticas y económicas que ponen a las personas en peligro de manera sistemática.

Creo que eso es lo realmente útil de todo esto y quizás un tipo de respuesta ha sido la competencia estructural. La primera persona a quien se le ocurrió ese término fue Jonathan Metzl en su libro The protest psychosis hace unos ocho años. No lo desarrolló como un campo todavía, pero dijo que quizás, en lugar de la competencia cultural, realmente necesitamos competencia estructural. Sabemos que estos procesos sociales son los que hacen que las personas estén más o menos 
enfermas de diferentes maneras $y$, desde ese momento, Jonathan Metzl en Vanderbilt, Helena Hansen en New York University, Kelly Knight en University of California-San Francisco, un grupo de personas que incluye a Joshua Neff y Shirley Strong, yo y otras personas en el grupo de trabajo de competencia estructural (Structural Competency Working Group), Sam Dubal y la gente de la Oregon Health \& Science University, junto a personas en Frankfurt, Alemania; Viena, Austria y en Sudáfrica y Australia hemos estado pensando: ¿cómo implementamos la competencia estructural?, ¿cómo se vería?, ¿cómo enseñar a los médicos que están tan entrenados en los modelos biológicos y comportamentales para pensar estructuralmente, imaginar la producción estructural de salud y respuestas o intervenciones estructurales?

Muchos de nosotros hemos estado trabajando en el desarrollo de capacitaciones, pensando cómo podría funcionar, ver si ayudan a los profesionales de la salud a interactuar de manera diferente con sus pacientes. $Y$ una cosa que hemos encontrado en el Structural Competency Working Group, aquí en el Bay Area, es que desarrollamos una capacitación de cuatro horas para estudiantes de medicina, pasantes y médicos. Y después de la primera capacitación, las personas que lo hicieron dijeron: "esto es muy importante", "me da un lenguaje para pensar sobre las cosas que he estado viendo", "me ayuda a verlas de manera diferente a como lo había hecho antes", y "sigo pensando en ello todo el tiempo". Pero también dijeron "me siento abrumado porque no estoy seguro de cómo responder". Entonces, cambiamos la capacitación y agregamos una sección importante de ejemplos de médicos, agentes de salud de diferentes comunidades, grupos de pacientes y de los vecindarios, que pudimos encontrar a lo largo de la historia, que hacen cosas que podríamos denominar intervenciones estructurales, que afectan la salud, para que pudieran pensar en ejemplos y ver que, a veces, las respuestas son individuales, como ayudar a un individuo a encontrar recursos para la vivienda o algo. Sin embargo, a veces, las intervenciones son colectivas: un barrio se reúne, o el Colectivo de Psicología Crítica de Tarapacá se reúne y apoya a las personas de Alto Hospicio después de que son expulsados violentamente o lo que sea. Si bien a menudo el cambio ocurre de manera colectiva, lo que hemos visto en las respuestas sobre la nueva versión de la capacitación es que las personas encontraron útiles el lenguaje y las ideas también para interactuar con sus pacientes individualmente. A menudo sienten más empatía o solidaridad con sus pacientes, en lugar de sentirse como "estoy trabajando muy duro para ti y sigues fumando, aunque te digo que no lo hagas y estoy muy frustrado contigo". En cambio, los médicos y los profesionales de la salud a menudo comienzan a sentir, o dicen que se han sentido más como "estás experimentando violencia estructural y estás vulnerable debido a estas estructuras sociales y las estructuras sociales me afectan también porque solo tengo diez o quince minutos para verte, y el sistema de seguros de salud en EEUU es un ejemplo muy claro de una estructura que es confusa e inútil para muchas personas". Entonces hay menos antagonismo y más "estamos juntos en el mismo barco". Y se han sentido menos abrumados, porque tienen ejemplos de médicos individuales y de comunidades y colectivos que han podido hacerlo.

James: voy a usar un ejemplo: la Clínica Martín-Baró. Es una clínica gratuita que solo está abierta y accesible para las personas los sábados y está ubicada en el Distrito de la Misión [San Francisco], el principal barrio latino. Las personas que mantienen y atienden la clínica son estudiantes de medicina de la University of California-San Francisco y universitarios y estudiantes de posgrado de la San Francisco State University en psicología, en estudios latinos, en trabajo social. No solo han creado una clínica a la que pueden acceder personas que no tienen seguro de salud, sino que también hacen actividades de divulgación en la comunidad para que las personas sepan que hay una clínica disponible para ellas. Proporcionan atención primaria de salud básica y han creado relaciones con farmacias locales y con el Hospital General de San Francisco, 
que se encuentra a pocas cuadras de distancia. Lo que han hecho es realmente establecer contactos e intentar crear vínculos viables con otros servicios hasta el punto en que, por ejemplo, dentro de la clínica, tienen lo que se llama el "orientador del paciente" [patient navigator]; es un defensor, una persona que trabaja allí, que acompaña al paciente a la farmacia, al hospital, a la clínica o al dentista. No solo asumen la responsabilidad de asegurarse de que los pacientes tengan citas, sino que también acudan a las citas. Van un paso más allá de la forma convencional en que pensamos acerca de la atención médica en la que vas, esperas 20 minutos o más, ingresas, te atienden entre 15 y 20 minutos, te dan una receta y te vas, $y$ te dicen que tal vez tengas una cita dentro de un mes, o te remiten a un especialista para el que tienes que hacer una llamada.

La práctica en la clínica [Clínica MartínBaró] es completamente diferente. Tiene una posición más de defensa del paciente y, con respecto a la población con la que trabajo (jornaleros latinos indocumentados), ha tenido una llegada maravillosa a ese grupo de jornaleros, generalmente mayores, que han estado en el país durante años y años y por alguna razón no pueden regresar a México, Nicaragua o Honduras. Tienen todo tipo de problemas, no tienen un ingreso estable, no tienen seguro de salud, $y$, sin embargo, la Clínica ha acudido a ellos, ha realizado actividades de divulgación. $\mathrm{Y}$ una buena parte de las personas que van a la Clínica son jornaleros indocumentados, personas que no tienen otras alternativas o lugares para obtener el tipo de servicios de salud que necesitan.

Seth: ¿Podrías decir algunas cosas más de dónde se está utilizando la vulnerabilidad estructural de manera útil?

James: Sí, hay un libro que saldrá a principios de 2019 y es de la School of Advanced Research sobre el cáncer y la vulnerabilidad estructural. Me convocaron para trabajar junto a varios antropólogos médicos y otros científicos sociales especializados en cáncer, para ver cómo podríamos movilizar y desarrollar la idea de vulnerabilidad estructural para pacientes y sobrevivientes de esta enfermedad. Es un grupo bastante notable de investigadores del cáncer que trabajan en Francia, en Puerto Rico, en Vietnam, y también aquí en EEUU, y que intentan determinar la experiencia de tener cáncer, de lidiar con él, con la terapia y el tratamiento necesario, y cómo podría ayudar la vulnerabilidad estructural a comprender la experiencia del cáncer.

Entonces, por ejemplo, una cosa que surge invariablemente es que, para muchos pacientes con cáncer y para los sobrevivientes, todo su mundo se invierte [is turned upside down], ya que, si están trabajando, tal vez tienen que comprometer el tipo de trabajo que hacen, recortar horas o directamente dejar el trabajo. La relación con su familia, las redes sociales en las que confían están pasando por una completa renegociación de cómo tratar a alguien que tiene cáncer. Dependiendo de si tiene seguro de salud y el tipo de seguro de salud que tiene, ¿Cubrirá el seguro el medicamento requerido? Pero, además, requieren diferentes tipos de tratamientos, quimioterapia, radiación, y todas las deficiencias que aparecen. Volviendo al Estado, ¿lo cubrirá Medicare? En Vietnam, ¿el Estado será realmente capaz de atender a las personas que tienen cáncer? Lo que hicimos con respecto al cáncer fue entender que el mundo social con el que tienen que lidiar los pacientes de cáncer está tan alterado, que requiere adoptar una perspectiva centrada en el paciente [patient centered perspective] de cómo ha cambiado su mundo. Para probar y descubrir las diferentes formas en que las personas pueden recibir ayuda para pasar por su tratamiento y, con suerte, poder sobrevivir y seguir adelante.

Les daré un ejemplo. Están Carolyn Sargent y Peter Benson que trabajaron con mujeres refugiadas malienses en Francia. En las mujeres refugiadas de Malí hubo una alta incidencia y prevalencia de cáncer de mama. En Francia, por lo general, no pueden hacer preguntas sobre la raza, y creo que se trata de una historia francesa de égalité, del espíritu igualitario, en el que no se pregunta ese tipo 
de cosas. Sin embargo, muchos epidemiólogos y muchos investigadores del cáncer se encontraron con la dificultad de poder estudiar cómo se agrupaba el cáncer en poblaciones particulares y aquí estamos tratando con una población que era principalmente de África Occidental y muchos de ellos eran indocumentados. El problema fue determinar cuál debería ser la intervención clínica, porque tenían cáncer, tenían acceso a la atención médica $y$, sin embargo, muchos se mostraron reacios a acudir a la atención médica... porque si se conociera su estado migratorio, incluso si pasan por el tratamiento, incluso si se curan, se los enviaría de inmediato a su país. Serían deportados.

Entonces, la pregunta se convirtió en ¿cómo podemos obtener profesionales de la salud capaces de reconocer ese tipo de situaciones y de documentarlas? ¿Cómo negociar una forma de proporcionar la atención y el tratamiento necesarios para las personas con las que estás tratando, sin poner en peligro su estatus familiar, su estatus laboral, su estatus de residencia? Y sigue siendo un desafío en Francia, que cuenta con clínicas de atención médica, médicos, enfermeras y trabajadores sociales especialmente progresistas. ¿Cómo puede ofrecer estos servicios de manera abierta, pero, al mismo tiempo, no poner en peligro el estado de las mismas personas a las que intenta ayudar?

Aquí es donde, una vez más, debemos pensar en la atención de la salud más allá de una intervención e intercambio puramente biomédicos, que integre también los determinantes sociales de la salud, pues es una práctica institucional que tiene ramificaciones sociales, tiene consecuencias sociales, así como las consecuencias personales individuales. Y la pregunta que surge es: ¿cuál es la responsabilidad de los profesionales de la salud al abordar, no solo los problemas biológicos que presentan las personas, sino todo lo demás que las rodea? Así que, una vez más, no sé si estoy respondiendo a tu pregunta.

Carlos: Sí, eso es interesante. Realmente se conecta con uno de los problemas profundos que me interesan y hemos hablado con
Seth. En mi país, existe una política de salud pública sobre la salud de los inmigrantes y, mirando la propuesta de la política desde el punto de vista de la vulnerabilidad estructural y la determinación política de los problemas de salud, descubrimos que uno de sus problemas profundos es que reduce el racismo a su dimensión interpersonal, desconociendo el racismo estructural. Entonces, quisiera escuchar tus ideas sobre cómo un marco de vulnerabilidad estructural puede contribuir a la formulación de mejores políticas públicas.

James: Pienso que, desde esta perspectiva, la salud pública es una ciencia subversiva. Si realmente fuera a asumir el desafío de hacer lo necesario para el bienestar y asegurarse de que tanto las personas como las poblaciones puedan lograr una mejor salud, la salud pública está en una posición extraordinaria para argumentar que debemos no solo tomar en serio los determinantes sociales de la salud, sino que realmente debemos repensar las políticas y las prácticas.

Sabemos qué es lo que contribuye a la mala salud y qué tiene que ser cambiado tanto en términos de vulnerabilidad estructural como de competencia estructural y, en ese sentido, debemos reconocer que los profesionales de la salud, en particular los médicos, tienen una autoridad médica extraordinaria. Con esa autoridad médica y el capital simbólico que tienen para usar si lo quisieran, no solo de forma individual, sino colectiva, por ejemplo, a través de la American Medical Association, deberían hablar de cómo está viviendo la gente, si las personas están obteniendo un sueldo viable para poder realmente cuidarse a sí mismas, averiguar por qué encontramos tremendos desiertos alimentarios en áreas urbanas, abordar qué es lo que debería considerarse como vivienda, etc. Pero, de hecho, no son entendidos como problemas de salud que competan a los médicos.

Y se trata de poder educar, creo que la población en general, la ciudadanía, debe comprender estas cosas desde el punto de vista de la salud pública y no estrictamente como cuestiones políticas que parecen desconectadas. 
Así que creo que, tomando la perspectiva de la vulnerabilidad estructural, podemos ver que no son solo los jornaleros indocumentados, o los refugiados malienses en Francia, son todas las personas que han estado sujetas a lo que yo llamo los "-ismos". Y aquí es donde interviene la violencia estructural. Los "ismos": racismo, sexismo, capacitismo [ableism], todas esas cosas se generan en poblaciones y deben abordarse adecuadamente. Y creo que las personas involucradas en la salud pública pueden abordar formidablemente estos problemas, sin moralizar o, incluso, sin necesidad de argumentos politizados; sino utilizando un argumento de la salud y una perspectiva de la salud, que contribuya a las consideraciones morales y políticas que también deben tenerse en cuenta.

Creo que desde una perspectiva médica y de salud, tienes el poder de acentuar cómo se deben tomar en cuenta las fuerzas sociales y estructurales; y se deben abordar como problemas en sí mismos, porque cuando no te enfrentas a ellos, estás generando aún más problemas de salud, de todos los tipos.

Carlos: He visto que aquí en Berkeley están interesados en lo que ha sido la discusión teórica y práctica en América Latina; específicamente, en la medicina social latinoamericana, así que, realmente, quisiera escuchar sobre ese interés en el marco de la discusión teórica y práctica que están haciendo aquí.

Seth: De cierta manera, me siento un poco avergonzado y creo que nosotros, en EEUU y en el mundo anglófono, estamos atrasados en contraste con la teorización latinoamericana de la determinación social de la salud. Aquí tendemos a hablar de los determinantes sociales de la salud y hay variables que las personas consideran, y es importante considerarlas, pero a menudo esas desigualdades, se dan por sentado que existen en el mundo. Luego, por lo general, en el mundo anglófono solo observamos los mecanismos entre las desigualdades, las jerarquías y los resultados y pensamos en cómo cambiar esos mecanismos, pero no pensamos en cuáles son los motores de la sociedad que están produciendo esas desigualdades y las diferencias demográficas que hacen que las personas se enfermen o mueran.

Entonces, a veces hemos hablado sobre los determinantes estructurales de los determinantes sociales de la salud, para que la gente dé un paso más, pero Jaime Breilh y otras personas de América Latina han escrito sobre la determinación social de la salud para hacernos pensar realmente quién es el dueño de los medios de producción y quién no, quién está siendo explotado y quién no, y cómo está racializado, o cómo se relaciona eso con los pueblos indígenas. Y todas esas preguntas creo que son realmente importantes. El movimiento que Jim Quesada, Philippe Bourgois y Laurie Hart y otros han estado haciendo sobre la vulnerabilidad estructural, está tratando de traer algo de esa conciencia de la determinación política y social de la salud en la que América Latina ha pensado mucho, más que el mundo anglófono, en cierto modo. $Y$, sobre todo, hacerlo de una manera que los clínicos, los profesionales de la salud y de la salud pública puedan comenzar a entenderlo y considerarlo como algo que importa.

Hay mucha gente en América Latina que ha estado pensando en estos temas de muchas maneras que son realmente útiles $y$, tal vez, de alguna forma, la vulnerabilidad estructural se ajusta a la antropología médica crítica y también a la medicina social, creo que encaja con esas otras ideas. Pero cuando pienso en lo que añade a una lente de determinación social, no sé exactamente. Desde mi punto de vista, la vulnerabilidad estructural se centra en la determinación social y política de la salud para enriquecer las ciencias de la salud en sí mismas, así como en desafiar las prácticas de salud institucional para implementar una verdadera medicina social. La vulnerabilidad estructural mejora la comprensión demográfica, individualizada, privatizada y conductualizada de la vulnerabilidad con un entendimiento de cómo las estructuras sociopolíticas y económicas sistemáticamente producen la vulnerabilidad en diferentes personas de diferentes maneras. Espero que esta importante apertura para 
comprender la determinación social de la salud lleve a un cambio en la educación de los profesionales de la salud, en la práctica de la medicina y la salud pública y, en última instancia, en la sociedad.

\section{AGRADECIMIENTOS}

Carlos Piñones Rivera agradece a Seth M. Holmes, James Quesada, Charles Briggs, Clara MantiniBriggs, Deborah Freedman Lustig, Nancy ScheperHughes, Michael Hughes, Shirley Strong y Denise Herd, por la acogida como visiting scholar en el Institute for the Study of Societal Issues del Berkeley Center for Social Medicine, el segundo semestre del año 2018. También al programa Fondecyt Postdoctorado de la Comisión Nacional de Investigación Científica y Tecnológica (Conicyt) por el financiamiento para la pasantía en cuyo contexto se realizó la entrevista (Proyecto 3180173 [20182020], "Itinerarios terapéuticos transfronterizos"). James Quesada agradece al National Institutes of Health, National Institute on Drug Abuse (NIH/ NIDA) por el financiamiento para el proyecto 1ROIAAOI7592-OIA2 "Structural-Environmental Factors, Alcohol and HIV Risk among Latino Migrant Laborers".

\section{REFERENCIAS BIBLIOGRÁFICAS}

1. Quesada J, Hart LK, Bourgois P. Structural vulnerability and health: Latino migrant laborers in the United States. Medical Anthropology. 2011;30(4):339-362. doi: 10.1080/01459740.2011.576725.

2. Villa-Torres L, González-Vázquez T, Fleming PJ, González-González EL, Infante-Xibille C, Chavez R, Barrington C. Transnationalism and health: A systematic literature review on the use of transnationalism in the study of the health practices and behaviors of migrants. Social Science and Medicine. 2017;183:70-79. doi: 10.1016/j. socscimed.2017.04.048.
3. Metzl JM, Roberts DE. Structural competency meets structural racism: race, politics, and the structure of medical knowledge. AMA Journal of Ethics. 2014;16(9):674-690. doi: 10.1001/virtualmentor.2014.16.9.spec1-1409.

4. Feagin J, Bennefield Z. Systemic racism and U.S. health care. Social Science \& Medicine. 2014;103:714. doi: 10.1016/j.socscimed.2013.09.006.

5. Ford $\mathrm{CL}$, Airhihenbuwa CO. Critical race theory, race equity, and public health: Toward antiracism praxis. American Journal of Public Health. 2010;100(Suppl 1):693-698. doi: 10.2105/ AJPH.2009.171058.

6. Ford $\mathrm{CL}$, Airhihenbuwa CO. The public health critical race methodology: Praxis for antiracism research. Social Science \& Medicine. 2010;71(8):13901398. doi: 10.1016/j.socscimed.2010.07.030.

7. Viruell-Fuentes EA, Miranda PY, Abdulrahim S. More than culture: Structural racism, intersectionality theory, and immigrant health. Social Science \& Medicine. 2012;75(12):2099-2106. doi: 10.1016/j.socscimed.2011.12.037.

8. Crenshaw K. Mapping the margins: Intersectionality, identity politics, and violence against women of color. Stanford Law Review. 1991;43(6):12411299. doi: 10.2307/1229039.

9. Bourgois P. Conjugated oppression: class and ethnicity among Guaymi and Kuna banana workers. American Ethnologist. 1988;15(2):328-348.

10. Neff J, Knight KR, Satterwhite S, Nelson N, Matthews J, Holmes SM. Teaching structure: A qualitative evaluation of a structural competency 
training for resident physicians. Journal of General Internal Medicine. 2017;32(4):430-433. doi: 10.1007/s11606-016-3924-7.

11. Bourgois P, Holmes SM, Sue K, Quesada J. Structural vulnerability: Operationalizing the concept to address health disparities in clinical care. Academic Medicine. 2017;92(3):299-307. doi: 10.1097/ACM.0000000000001294.

12. Metzl JM, Hansen H. Structural competency: Theorizing a new medical engagement with stigma and inequality. Social Science \& Medicine. 2014;103:126-133. doi: 10.1016/j.socscimed. 2013.06.032.

13. Hernández-Rosete Martínez D, Sánchez Hernández G, Pelcastre Villafuerte B, Juárez Ramírez C. Del riesgo a la vulnerabilidad: Bases metodológicas para comprender la relación entre violencia sexual e infección por VIH/ITS en migrantes clandestinos. Salud Mental. 2005;28(5):20-26.

\section{FORMA DE CITAR}

Piñones Rivera C, Quesada J, Holmes SM. La vulnerabilidad estructural y las nuevas perspectivas en medicina social sobre la salud de los migrantes: entrevista a James Quesada y Seth M. Holmes. Salud Colectiva. 2019;15:e2146. doi: 10.18294/sc.2019.2146.

Recibido: 7 de febrero de 2019 | Aprobado: 15 de abril de 2019

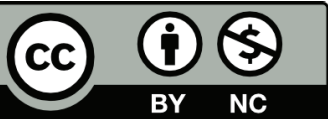

Esta obra está bajo una licencia de Creative Commons Reconocimiento-NoComercial 4.0 Internacional. Reconocimiento - Permite copiar, distribuir y comunicar públicamente la obra. A cambio, se debe reconocer y citar al autor original. No Comercial - Esta obra no puede ser utilizada con finalidades comerciales, a menos que se obtenga el permiso. 